This document is the unedited Author's version of a Submitted Work that was subsequently accepted for publication in Analytical Chemistry, copyright (c) American Chemical Society after peer review. To access the final edited and published work see: https://dx. doi.org/10.1021/acs. analchem.0c02619. 


\title{
One-step immobilization of antibodies and DNA on gold sensor sur- faces via poly-Adenine oligonucleotide approach
}

\author{
Cesar S. Huertas $\stackrel{\mathrm{a}, \mathrm{b}, *, *, \text { Maria Soler }^{\mathrm{a}, \mathrm{c}, \$}, \text { M.-Carmen Estevez }}{\mathrm{a}}$, and Laura M. Lechuga ${ }^{\mathrm{a}}$ \\ ${ }^{a}$ Nanobiosensors and Bioanalytical Applications group, Catalan Institute of Nanoscience and Nanotechnology (ICN2), \\ CSIC, CIBER-BBN and BIST, Barcelona, Spain. \\ ${ }^{\mathrm{b}}$ Integrated Photonics and Applications Centre, School of Engineering, RMIT University, Melbourne, Australia. \\ ${ }^{\mathrm{c}}$ Networking Research Center on Bioengineering, Biomaterials and Nanomedicine (CIBER-BBN), Spain. \\ * corresponding author: cesar.sanchez.huertas@rmit.edu.au
}

KEYWORDS: optical biosensor, biofunctionalization, antibody, DNA, surface chemistry, surface plasmon resonance

\begin{abstract}
Label-free plasmonic biosensors have demonstrated promising capabilities as analytical tools for the detection of virtually any type of biomarker. They are presented as good candidates for precision diagnostics since they offer highly sensitive, cost-effective solutions that can be used in any clinical or laboratory setting without the need for specialized trainees. However, different surface functionalization protocols are required depending on the nature of the biorecognition element, limiting their capabilities for integrated multibiomarker detection. Here, we present a simple, yet efficient one-step immobilization approach that is common for both DNA probes and antibodies. Our immobilization approach relies on the incorporation of polyA blocks in both nucleic acid probes and antibodies, which specifically interact with the gold surface with sufficient strength to generate dense and highly ordered monolayers. We have demonstrated excellent performance of our universal functionalization method, showing limits of detection and quantification in the pM-nM range. Moreover, it was able to reduce up to $50 \%$ the background signal from undiluted serum samples compared to conventional methods, demonstrating the immense potential of this strategy for the direct analysis of human biofluids, essential for rapid point-of-care diagnostics. The polyA-based immobilization approach is a promising alternative for the biofunctionalization of microarray-format sensors and their application to multiplexed diagnostic assays.
\end{abstract}

Precision diagnostics is a growing field seeking to transform healthcare by providing comprehensive, insightful and accurate clinical information of patients. ${ }^{1}$ Its implementation can foster individualized disease management and, ultimately, improve the patient outcome and quality of life. To meet these requirements, there is a need to deploy advanced tools that can inform about the patient molecular fingerprint, including variations in their genome, epigenome, transcriptome, proteome and metabolome, which will allow to relate them with a concrete disease. Ideally, these tools should afford multimarker detection with ultra-high sensitivity and specificity to shed light about the interplay between different biomarkers of diverse nature and recognize specific patterns that can inform about tailored treatments. ${ }^{2}$

Biosensors are analytical devices that can employ multiple biorecognition elements, such as antibodies or DNA probes, for the specific detection of target analytes in a sample, such as proteins, nucleic acids, small molecules or even whole cells, like viruses or bacteria. ${ }^{3}$ Optical biosensors explore the interaction of the analyte with electromagnetic fields and have given rise to some of the most sensitive, robust, and versatile analytical devices. Particularly, plasmonic and nanoplasmonic biosensors are nowadays positioned at the forefront of optical technologies for the achievement of new powerful devices with potential applications not only in clinical diagnostics, biomedical and pharmacological studies, but also in food safety, and environmental monitoring. ${ }^{3}$ The landmark of optical biosensors is the Surface Plasmon Resonance (SPR) system. SPR biosensors are based on the excitation of the so-called surface plasmon polaritons, which are coherent oscillations of conductive electrons at the surface of a nanometer-thick layer of certain metals, being gold the most widely employed. These plasmons generate an evanescent field at the metal surface that penetrates and exponentially decays into the surrounding dielectric medium reaching a few hundreds of nanometers (100$500 \mathrm{~nm}$ ). A change of mass or composition of the dielectric within the evanescent field produces a change of the refractive index (RI) that will be, in turn, transduced into a change in the light properties, such as intensity, wavelength, or phase. This sensing principle therefore enables the direct quantification of specific analytes as a one-step assay, without need of labelling and in real time. ${ }^{4}$

The label-free analysis capability of plasmonic biosensors is certainly one of their best assets, but it also implies certain limitations. The overall analytical sensitivity and specificity of these systems ultimately depend on the sensor surface biofunctionalization. An optimum immobilization of the biorecognition elements on the gold layer must ensure their correct orientation, optimal coverage density and high stability, and provide antifouling properties that prevent the non-specific bind- 
ing of non-relevant molecules. The latter is especially important when dealing with complex biological samples, like blood serum or plasma, since the adsorption of matrix components onto the sensor surface can lead to false-positive signals.

Over the last years, a myriad of sensor biofunctionalization strategies have been described. ${ }^{2,5}$ In the case of antibodies, as the most widely used biorecognition element, a common approach is the covalent binding to a self-assembled monolayer (SAM) of functional alkanethiol molecules through the amineterminal residues. ${ }^{6}$ This well-known methodology provides a robust biomolecule attachment and certain coverage of the remaining gold surface. However, the antibodies are immobilized in random orientation, so hindering part of the antigen binding sites (Fab regions). In order to control antibody orientation, one can make use of affinity ligands such as the proteins $\mathrm{A}$ or $\mathrm{G}$ that bind to the $\mathrm{Fc}$ region of antibodies, or the biotin-avidin pair, when the antibody is biotinylated through the carbohydrate $\mathrm{Fc}$ chains..$^{7-9}$ Other strategies propose antibody fragmentation via disulfide bond reduction for direct chemisorption of the resultant thiols (-SH), or the immobilization of only Fab chains. ${ }^{10}$ All these stepwise methodologies are usually employed in immunosensor development and have demonstrated undeniable advantages over simple, direct but low reproducible physical adsorption of the antibodies. However, it is critical to perform, in each case, an in-depth optimization of every procedure step to select the correct SAM composition, activation times, concentration of affinity ligands, the antibody-tag conjugation, or fragmentation reaction times. In the case of DNA, the possibility of obtaining synthetic probes of high purity containing the desired sequence and including an end-terminal functional group, greatly facilitates the surface biofunctionalization. ${ }^{11}$ For gold surfaces, the most common and simple strategy is to use the DNA oligonucleotide sequence with a thiol (-SH) group for direct chemisorption on gold. To control the DNA probe density and minimize steric hindrance effects, it is frequent to include alkanethiol molecules as lateral spacers, or to immobilize the DNA strand onto a SAM, similarly to antibody immobilization. ${ }^{2}$ Likewise, all these surface chemistry strategies must be carefully studied and optimized during biosensor development to achieve the best analytical performance.

The major challenge is found on the road of technology transfer and implementation of these biosensors in the clinical field. The implementation of different biofunctionalization protocols involving different steps for both, protein and DNA biomarker detection, can be complex and cumbersome, limiting the capabilities of plasmonic biosensors. Ideally, the sensor biofunctionalization should be an easy and direct procedure that can be automated, highly reproducible, and ensuring maximum bioreceptor activity. In this work, we propose a surface biofunctionalization approach for gold-based sensors that enables one-step and rapid immobilization of antibodies and DNA probes in an oriented manner, with controlled density and distribution, and antifouling properties. Our strategy relies on the preferential and remarkably high affinity of adenine (A) for gold, and the dramatic relative difference with thymine (T) (Figure 1A). Indeed, DNA nucleotides affinities for gold follow the order $\mathrm{A}>\mathrm{G} \approx \mathrm{C}>\mathrm{T} .{ }^{12}$ It has been shown that strands of poly $\mathrm{A}_{\mathrm{m}}$-poly $\mathrm{T}_{\mathrm{n}}$ attach to gold surfaces adopting an L-shaped conformation, where the polyA tail binds completely flat to the surface while the polyT chain extends away from the surface, vertically oriented. ${ }^{13,14}$ Contrary to what might be expected, the hairpin conformation of self- complementary poly $\mathrm{A}_{\mathrm{m}}$ - poly $_{\mathrm{n}}$ oligonucleotides in solution is disrupted in presence of gold, resulting in close-saturation coverage of the metal sensor surface by the polyA blocks. ${ }^{14}$ We have incorporated the poly $\mathrm{A}_{\mathrm{m}}-$ poly $_{\mathrm{n}}$ tails to both DNA probes and antibodies and optimized the methodology for the direct and label-free detection of clinically relevant biomarkers (Figure 1B). By varying the polyA length, it is possible to deterministically control the probe density and distribution, while the polyT block provides the upright orientation. We have studied the analytical performance of our approach for SPR biosensing, comparing it with other traditional biofunctionalization strategies, evaluating the antifouling properties for the analysis of clinical samples (i.e., blood serum), and exploring the capabilities for sensor surface regeneration and reusability. Our versatile polyA approach is compatible with conventional laboratory procedures, so it could be implemented in the research and industrial production lines of antibodies and DNA probes, even including other protein receptors, enzymes, and aptamers. ${ }^{15-17}$ Also, it could be applied for other gold-based sensor and biosensor technologies, such as nanoplasmonic arrays or colloidal nanoparticles, electrochemical or piezoelectric sensors, among others.

Figure 1. PolyA immobilization strategy. (A) Scheme of the pro-
A

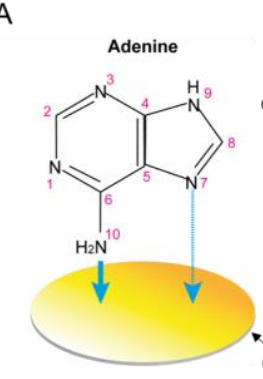

B

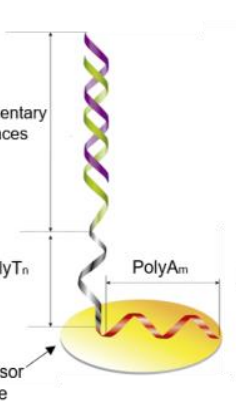

Antigen

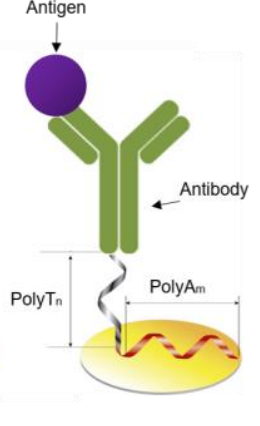

posed mechanism for adenine adsorption on gold surfaces: major interaction is attributed to coordination to the metal by the $\mathrm{N}$ atoms of the amine group and the $\mathrm{N}_{7}$ atom. (B) DNA probe and antibody immobilized on the gold sensor surface in an L-shape conformation.

\section{EXPERIMENTAL DETAILS}

Chemical and Biological Reagents and Buffers. The list of chemical and biological reagents, buffers and solvents used in this work is provided in the Supporting Information.

SPR Biosensor. The SPR studies have been performed by the ICTS "NANBIOSIS", more specifically at the Unit of Biodeposition and Biodetection (U4) of CIBER in Bioengineering, Biomaterials \& Nanomedicine (CIBER-BBN) at ICN2 facilities. The SPR biosensor device is based on the Kretschmann configuration and monitors RI changes in real time. A ppolarized light of $670 \mathrm{~nm}$ from a laser source is divided in two identical beams focused on the backside of the gold sensor chip (glass surface coated with $1.5 \mathrm{~nm}$ of titanium and $48 \mathrm{~nm}$ of gold, $10 \times 10 \times 0.3 \mathrm{~mm}^{3}$, SSens, Enschede, The Netherlands). Measurements are performed at a fixed angle of incidence. Variations of the RI due to the biointeraction events occurring at the sensor surface are detected as changes in the reflected light intensity by a multielement photodiode. The flow system consists of two flow cells ( $300 \mathrm{~nL}$ each) for independent analysis. The device incorporates all optical, electronic and fluidic components necessary to operate autonomously. Sensorgrams reproduce the binding events by monitoring the increase (or 
decrease in case of unbinding events) of the intensity of the reflected light ( $\Delta$ Reflectivity $(\%), \Delta \mathrm{R}(\%))$ vs. time (seconds, $\mathrm{s})$. This change in the intensity of the reflected light is directly related to changes in the RI of the dielectric medium caused by mass changes on the metallic sensor surface.

PolyA-DNA probes immobilization. Formation of monolayers of polyA-DNA probes was carried in situ at $1 \mu \mathrm{M}$ concentration in $\mathrm{CaCl}_{2}$-TE buffer. Different polyA $\mathrm{A}_{\mathrm{m}}$ block lengths were employed, where $m$ is the number of adenine nucleotides $(\mathrm{m}=7,15)$, i.e. PolyA $\mathrm{A}_{7}$ and PolyA $_{15}$. Several combinations of the probes presenting the two different block lengths at different molar ratios (i.e polyA $7 /$ poly $_{15}=1: 0,0: 1,1: 1,1: 2$ and 2:1) were tested. $\mathrm{H}_{2} \mathrm{O}$-DEPC was selected as running buffer for the immobilization procedure at a constant rate of 12 $\mu \mathrm{L} / \mathrm{min}$, approximately. Conventional immobilization strategies (thiol chemisorption and amine covalent coupling) are described in the Supporting Information.

DNA hybridization and biosensor regeneration. Samples containing Fas567 target sequence were injected into the biosensor at a $15 \mu \mathrm{L} / \mathrm{min}$ rate. Hybridization with their complementary DNA probes immobilized on the gold sensor surface was monitored in real-time by a custom-made LabVIEW Software. These samples were dissolved in the hybridization buffer $(5 \times \mathrm{SSC}-0.45 \mathrm{M} \mathrm{NaCl}, 0.045 \mathrm{M}$ sodium citrate- with $45 \%$ formamide). Target-probe interactions were disrupted by using $50 \%$ formamide in aqueous solution. A calibration curve was obtained for polyA-DNA probe by triplicate measurements of Fas567 at different concentrations (2.5, 5, 10, 20, 30, and $50 \mathrm{nM}$ ). The mean and the standard deviation (SD) of each concentration were plotted versus the target concentration and fitted to a saturation binding model. Limit of Detection (LOD) was calculated as the concentration corresponding to the blank signal plus three times its SD, while the Limit of Quantification (LOQ) was determined as the concentration corresponding to the minimum measurable signal, set as the blank plus 10 times SD.

PolyA-Antibody conjugation and immobilization. Monoclonal anti-CRP antibodies (Ab) were conjugated to $\mathrm{NH}_{2}-$ poly $_{26}$-poly $\mathrm{A}_{15}$ oligonucleotides via amine coupling (random orientation) and via aldehyde coupling (controlled orientation). For random conjugation we used bis(sulfosuccinimidyl) suberate (BS3) crosslinker. Antibodies $(1 \mathrm{mg} / \mathrm{mL})$ were diluted in $10 \mathrm{mM}$ PBS pH 7.5 and mixed with BS3 at 20-fold molar concentration. Reaction mixture was incubated at room temperature (RT) for 30 minutes and quenched with $50 \mathrm{mM}$ Tris buffer and purified using $30 \mathrm{~K}$ centrifugal filter units (Amicon Ultra, Merck Life Science S.L.U., Spain). BS3modified antibodies were mixed with the polyA tail $(1: 2$ Ab/oligonucleotide) and incubated for $2 \mathrm{~h}$ at RT in gentle agitation. The mixture was finally purified with $30 \mathrm{~K}$ centrifugal filter units and buffer was changed to $10 \mathrm{mM}$ PBS. For controlled orientation conjugation (see Figure S3 in the Supporting Information for the general scheme), anti-CRP antibodies $(1 \mathrm{mg} / \mathrm{mL})$ were first oxidized by incubation with $10 \mathrm{mM}$ $\mathrm{NaIO}_{4}$ in acetate buffer for $30 \mathrm{~min}$ at $\mathrm{RT}$ in dark conditions. Then, the antibodies were purified using $30 \mathrm{~K}$ centrifugal filter units and buffer was switched to $100 \mathrm{mM}$ bicarbonate buffer $\mathrm{pH}$ 6.0. In parallel, oligonucleotide modification with succinimidyl-6-hydrazino-nicotinamide (s-HyNic) crosslinker was carried out following supplier instructions: $1 \mathrm{mM}$ oligonucleotide was dissolved in $100 \mathrm{mM}$ bicarbonate buffer $\mathrm{pH}$ 8.0, and $200 \mathrm{mM}$ s-HyNic was dissolved in anhydrous dimethylfor- mamide (DMF). 20 molar equivalents of s-HyNic solution were added to the oligonucleotide solution, keeping the DMF percentage below $5 \%$. The mixture was incubated for $1.5 \mathrm{~h}$ at $\mathrm{RT}$, purified using $3 \mathrm{~K}$ centrifugal filter units and desalted to $100 \mathrm{mM}$ bicarbonate buffer $\mathrm{pH}$ 6.0. For conjugation, both solutions were mixed (1:2 Ab/oligonucleotide) and aniline $(100 \mathrm{mM})$ was added as catalyst. The mixture was incubated with gentle agitation for $2 \mathrm{~h}$, at RT, purified using $30 \mathrm{~K}$ centrifugal filter units, and buffer was switched to $10 \mathrm{mM}$ PBS. Characterization was carried out by spectroscopic determination using a UV Cary 4000 spectrometer measuring absorbance at $\lambda=354 \mathrm{~nm}$. The final concentration of the polyA-Ab conjugates was also determined by measuring absorbance of antibodies at $\lambda=280 \mathrm{~nm}$. Immobilization of the polyA-Ab conjugates was carried out in flow by injecting a $50 \mu \mathrm{g} / \mathrm{mL}$ solution diluted in $\mathrm{CaCl}_{2}$-TE buffer at $15 \mu \mathrm{L} / \mathrm{min}$. Conventional immobilization strategies (i.e physical adsorption and covalent coupling) are described in the Supporting Information.

Protein detection and biosensor regeneration. CRP protein samples in PBS were flowed over the biofunctionalized sensor surface at $15 \mu \mathrm{L} / \mathrm{min}$ flow rate and the response was monitored in real time. After each sample, regeneration of the surface was achieved by injecting $\mathrm{HCl} 5 \mathrm{mM}$ at $50 \mu \mathrm{L} / \mathrm{min}$. Calibration curves were obtained by triplicate measurements of CRP at different concentrations (50 - $2000 \mathrm{ng} / \mathrm{mL})$. The mean and the standard deviation (SD) of each concentration were plotted versus the target concentration and fitted to a saturation binding model. Limit of Detection (LOD) was calculated as the concentration corresponding to the blank signal plus three times its SD, while the Limit of Quantification (LOQ) was determined as the concentration corresponding to the minimum measurable signal, set as the blank plus 10 times SD.

Antifouling evaluation. The fouling effect was assessed for different monolayers of DNA (polyA-DNA, chemisorbed SHDNA, covalently attached $\mathrm{NH}_{2}$-DNA over a $\mathrm{MHDA} / \mathrm{MUOH}$ $\mathrm{SAM}$ ) and antibodies monolayers (polyA-Ab, Ab physical adsorption, covalently attached $\mathrm{Ab}$ over a MHDA/MUOH SAM). $250 \mu \mathrm{L}$ of $100 \%$ commercial Human Serum (SigmaAldrich, Steinhem, Germany) samples were flowed at a 15 $\mu \mathrm{L} / \mathrm{min}$ rate over the sensor surface. After that, serum was washed away by PBS running buffer. Shifts in the SPR sensor response baseline were measured to determine the background signal generated in each monolayer by the non-specific contact of the different components of the serum sample.

\section{RESULTS}

Our investigation on polyA-based sensor biofunctionalization approach for multimarker detection have included four main aspects: the design and preparation of polyA-conjugated DNA probes and antibodies, the study on the sensitivity and specificity of this strategy for direct and label-free detection of clinically relevant nucleic acid and protein biomarkers, the evaluation of the stability, reproducibility and reusability of the biosensor, and the antifouling properties for human biofluid analysis.

Design and preparation of polyA-conjugated DNA probes. The length of the homonucleotide polyA tail determines both the immobilization stability and the bioreceptor density and distribution. Likewise, the polyT block serves as vertical spacer to ensure the upright orientation and enough distance to the surface for efficient capture of the target analyte. In previous works, poly $\mathrm{A}_{15}$ tails were pointed to provide 
the best coverage and stability for DNA immobilization, and they were combined with additional poly $A_{k}$ spacers to control the density and target accessibility. ${ }^{14}$ However, the hybridization yields notably decreased in all cases when introducing poly $_{\mathrm{k}}$ spacers with different lengths (i.e. $\mathrm{k}=7,15$ or 25 ) and at increasing concentrations of these spacers. The introduction of polyA spacers effectively reduces the probe density at the expense of reducing the target binding capacity as well. In order to avoid dramatic changes in probe density and have a better probe density control, instead of using polyA sequences as spacers, we have designed two DNA probes including two different lengths of poly $A_{m}$ tails ( $m$ being 7 or 15). We hypothesize that, if we combine probes with different polyA lengths, we can fine-tune more precisely the probe density and, therefore, increase the accessibility of the target without compromising the number of probes available at the gold sensor surface. Each probe includes a poly $\mathrm{T}_{15}$ sequence to promote the upright conformation of the probes in an L-shaped conformation (Table S1). ${ }^{14}$

Biosensing performance of polyA-immobilized DNA probes. To demonstrate our biofunctionalization approach, we have selected Fas gene 567 mRNA as a model. Fas gene is involved in critical cell regulation and apoptotic pathways and has been demonstrated to be a good biomarker for early cancer diagnosis. Our previous work showed the excellent analytical performance of an SPR biosensor specific for this biomarker using SH-DNA probes. ${ }^{18,19}$ Thus, we will use it as a reference to compare with the polyA strategy. In addition, we also explored the analytical performance of DNA monolayers generated with MHDA/MUOH alkanethiols (for functionalization methods see Supporting Information). To study the analytical behavior of polyA-DNA probes, we monitored the immobilization process in real-time via in situ immobilization (i.e by tracking the SPR reflectance intensity shift in $\% \Delta \mathrm{R}$ during the probe injection). Several parameters were optimized to ensure a proper surface coverage and to provide a good probe density for an adequate accessibility of the target while minimizing non-specific adsorptions. $\mathrm{CaCl}_{2}$-TE buffer was employed for polyA grafting on the gold sensor chip, since it rendered the best immobilization signal (see Fig. S1). In addition, we compared the one-step poly-A immobilization strategy performance with the conventional thiol-chemistry based methodology and with the MHDA/MUOH monolayer. For that, we employed $1 \mu \mathrm{M}$ of polyA-DNA, SH-DNA and $\mathrm{NH}_{2}$-DNA probes respectively. As can be appreciated in Figure S2, immobilization of polyA-DNA probes provided a high sensor response, which was comparable to that obtained with $\mathrm{SH}$ DNA probes. $\mathrm{NH}_{2}$-DNA probes produced a lower sensor response, which could be explained by the low density of carboxylic groups available on the SAM monolayer. Next, to obtain the maximum hybridization yield, we evaluated the hybridization performances with the two selected poly $\mathrm{A}_{\mathrm{m}}$ block lengths, i.e. poly $\mathrm{A}_{7}$ and poly $\mathrm{A}_{15}$. Likewise, we tested combination of the probes with the two block lengths at different polyA $_{7} /$ polyA $_{15}$ molar ratios $(1: 0,0: 1,1: 1,1: 2$ and 2:1). All tested ratio provided similar immobilization signals, demonstrating that the immobilization efficiency was the same regardless of the length of the polyA block or their configuration (data not shown). We employed synthetic DNA sequences homologous to Fas567 mRNA (Table S1). Figure 2A shows a set of SPR measurements that quantitatively examines the effect in the hybridization efficiency of the two different polyA probes employed, either alone or co-immobilized at differ- ent ratios. Longer polyA $A_{m}$ blocks $(m=15)$ led to higher SPR responses, demonstrating a better accessibility of the targets. However, the intensity of the signal was largely improved by using a mix of the two polyA lengths, achieving the most suitable conditions at a 2:1 molar proportion of polyA $_{7} /$ polyA $_{15}$. These results confirm our hypothesis that a combination of different polyA tail lengths help controlling the density and accessibility of DNA probes on the gold surface in a more accurate and precise way, which may allow to further improve the biosensor performance.

For a close comparison of the analytical performance of the 2:1 polyA-DNA monolayer with the $\mathrm{SH}-\mathrm{DNA}$ and $\mathrm{NH}_{2}$-DNA approaches, we obtained a calibration curve by measuring different concentrations of Fas567 in triplicate, ranging from 0 to $50 \mathrm{nM}$, diluted in the hybridization buffer $(5 \mathrm{x}$ SSC $+45 \%$ FA). As can be seen in Figure 2B, increasing concentrations of Fas567 led to increasing SPR sensor responses. As a control, we employed a synthetic Fas 57 DNA sequence, which is the DNA homologous sequence of Fas 57 mRNA (Table S1). Fas 57 mRNA is generated from the same Fas mRNA precursor by a process called alternative splicing. This Fas isoform shares half of its sequence with Fas567 mRNA and, therefore, can be prone to cross-hybridization with our polyA-based Fas567 probe. As can be observed in Figure 2B, Fas57 generated negligible SPR response at different concentrations (from 5 to $50 \mathrm{nM}$ ), proving the specificity of our biosensor towards Fas567. To compare the polyA-DNA sensor performance, we calculated different analytical figures of merit, including limit of detection (LOD), and limit of quantification (LOQ). Using the polyA-DNA monolayer, the sensor achieved a LOD of $557 \mathrm{pM}$ and a LOQ of $1.9 \mathrm{nM}$. These results are very comparable with the ones obtained with the SH-DNA monolayer (LOD of $863 \mathrm{pM}$ and a LOQ of $3 \mathrm{nM}$ ) and the MHDA/MUOH monolayer (LOD of $698 \mathrm{pM}$ and a LOQ of $2.5 \mathrm{nM}$ ) using the same detection conditions. These results demonstrate that, although polyA blocks do not attach covalently to the gold sensor surface, it can render analogue biosensor performance to the conventional widely-used approaches, suggesting it as a good alternative for the generation of DNA monolayers.

Preparation of polyA-conjugated antibodies. Unlike DNA probes, which can be synthesized with the desired sequence and functional reactive groups, antibodies are biologically produced, therefore they must be conjugated to tags afterwards employing chemical crosslinkers. Conjugation of polyA tails to antibodies was carried out in two ways: via amine-terminal groups of the Lys present in the entire structure, which and leads to a random antibody orientation; and via carbohydrate side chains, for an end-on antibody orientation. In order to increase the flexibility and movement of the antibodies, and to ensure enough vertical and lateral spacing, we considered only the longer polyA sequence with $15 \mathrm{nu}-$ cleotides and a longer polyT sequence of 26 nucleotides (pol$\mathrm{yT}_{26}$ ) according to previous studies. ${ }^{20}$ In both cases, aminefunctional polyA oligonucleotide strands were employed $\left(\mathrm{NH}_{2}\right.$-poly $\mathrm{T}_{26}$-poly $\left.\mathrm{A}_{15}\right)$, following standard conjugation procedures described elsewhere (Figure S3) ${ }^{21,22}$ For non-oriented polyA-Ab conjugation we employed a homobifunctional amine crosslinker: bis(sulfosuccinimidyl)suberate (BS3). This crosslinker contains a N-hydroxysuccinimide (NHS) ester at each end, separated by an 8-carbon chain. The NHS ester readily reacts with terminal $\mathrm{NH}_{2}$ groups of lateral lysine chains, which are randomly distributed and available in antibodies. 
The other NHS ester binds the $\mathrm{NH}_{2}$-polyT $\mathrm{T}_{26}$-polyA $\mathrm{A}_{15}$ tail. This procedure is well-established and provide high conjugation yields, but it may result in chemical modification of the antigen binding sites (Fab regions) of antibodies therefore reducing their binding capability. ${ }^{21,23}$ For oriented polyA-Ab conjugation, we took advantage of the carbohydrate moieties mainly expressed on the Fc region of antibodies. The strategy essentially consists in creating active aldehyde (CHO) groups by mild oxidation of the cis-diols of carbohydrates. The $\mathrm{CHO}$ groups are highly reactive and can directly bind primary amines. However, direct binding of primary amines to activated aldehyde groups leads to weak and unstable bonds; instead, the terminal amine group of hydrazides is strongly nucleophilic and spontaneously reacts with aldehydes to form stable hydrazone bonds. ${ }^{24}$

To achieve that, we employed a heterobifunctional crosslinker: succinimidyl-6-hydrazino-nicotinamide (s-HyNic), that reacts with primary amines of the polyA oligonucleotide via NHS ester and introduces a reactive hydrazine group for the binding to the $\mathrm{CHO}$-activated antibodies. ${ }^{22} \mathrm{With}$ this procedure, the polyA tail is linked exclusively to the constant region of antibodies, which would be immobilized in an end-on configuration, leaving the antigen binding sites free and available for analyte detection. The conjugation reaction was traced and quantified by UV-spectrometry of the bis-arylhydrazone groups, which exhibits a characteristic absorbance peak at 354 nm (Figure S3). Determination of the absorbance in the resulting conjugate allows calculating the molar substitution ratio (MSR), set as the relative concentration of the bisarylhydrazone group per total antibody concentration. We obtained a MSR of 1.84, which means a total yield of the reaction of approximately $75 \%$. The applied procedure renders in a highly efficient polyA-Ab conjugation, and the resulting antibodies in solution were ready to be directly immobilized onto gold sensor surfaces.

Biosensing performance of polyA-immobilized antibodies. To assess the performance of polyA-Ab immobilization approach we selected the C-reactive protein (CRP) as a target biomarker model. This protein is a reference clinical biomarker for the detection of inflammatory procedures, such as infections, cardiac disorders, or some solid cancers (ref). Often, the quantification of CRP alone does not provide accurate diagnosis information, but it should be included in biomarker panels in combination with other proteins and nucleic acids to identify the origin of the disease. We compared the immobilization and detection efficiencies of the polyA approach with common antibody biofunctionalization procedures: physical adsorption and covalent binding to an alkanethiol SAM (MHDA/MUOH 1:20). ${ }^{9}$ Further, we evaluated both oriented and non-oriented conjugation strategies in terms of assay sensitivity. Figure 3A shows the antibody immobilization signals (at $50 \mu \mathrm{g} / \mathrm{mL}$ ) obtained with the SPR biosensor for the different biofunctionalization strategies. As expected, polyA-Ab conjugates produced similar responses than physical adsorption of antibodies onto the gold surface, which indicates a high coverage of the sensor. Covalent binding of antibodies to an alkanethiol SAM via carbodiimide chemistry renders lower antibody coverage, probably due to lower chemical reaction yields. But to accurately evaluate different biofunctionalization strategies, it is necessary to compare the detection performance in terms of sensitivity and specificity. We obtained standard calibration curves for the direct detection of CRP over a wide range of concentrations $(50-2000 \mathrm{ng} / \mathrm{mL})$ with
anti-CRP antibodies immobilized through the four different strategies (Figure 3B). Besides, we confirmed the detection specificity with negative control proteins (BSA). The best analytical performance in terms of sensitivity and detectability was obtained with the oriented polyA-Ab immobilization, with a LOD around $2 \mathrm{ng} / \mathrm{mL}$, and LOQ around $50 \mathrm{ng} / \mathrm{mL}$. Nonoriented immobilizations either with polyA tail or via covalent binding showed similar results in terms of detectability (LOD non-oriented polyA $=11 \mathrm{ng} / \mathrm{mL}$; LOD covalent binding $=7$ $\mathrm{ng} / \mathrm{mL}$ ) and quantification (LOQ non-oriented polyA $=36$ $\mathrm{ng} / \mathrm{mL}$; LOQ covalent binding $=72 \mathrm{ng} / \mathrm{mL}$ ), although the nonoriented polyA-Ab had a larger dynamic range, associated with the higher density of antibodies immobilized. On the contrary, physical adsorption strategy showed the poorest efficiency, with a LOD of $72 \mathrm{ng} / \mathrm{mL}$, a LOQ of $258 \mathrm{ng} / \mathrm{mL}$, and a very prompt saturation at relatively low biomarker concentrations. This can be explained by possible antibody denaturation in direct contact with the gold surface and absolute no-control of the orientation that hinder antigen binding, despite of the high surface coverage. These results demonstrate that the polyA-based biofunctionalization strategy is highly advantageous for antibody immobilization since it enables end-on orientation control as well as providing certain mobility to the antibodies, therefore maximizing the antigen binding capacity.

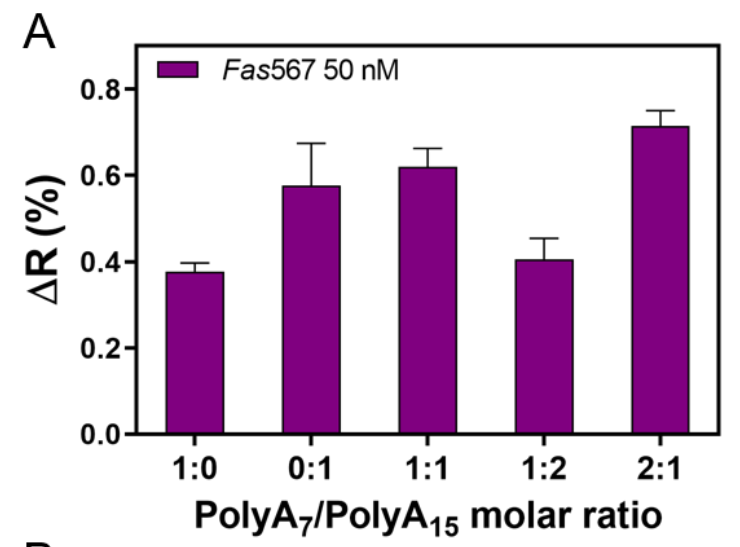

B

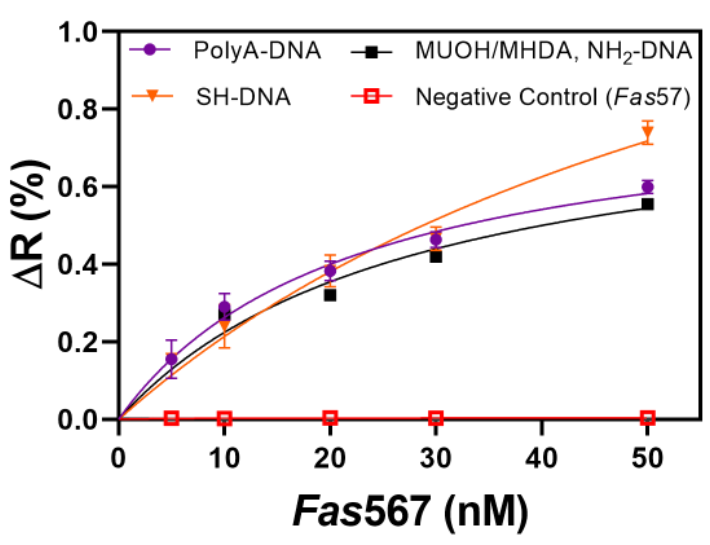

Figure 2. PolyA-DNA probe assay. (A) Hybridization signals of Fas567 in different polyA7/polyA 15 DNA-probe molar ratios. (B) Fas567 calibration curve for polyA-DNA monolayer, SH-DNA monolayer and MHDA/MUOH $\mathrm{NH}_{2}$-DNA monolayer. Solid line corresponds to the non-linear fit of the calibration curves. All data show mean \pm SD of triplicate measurements 
A

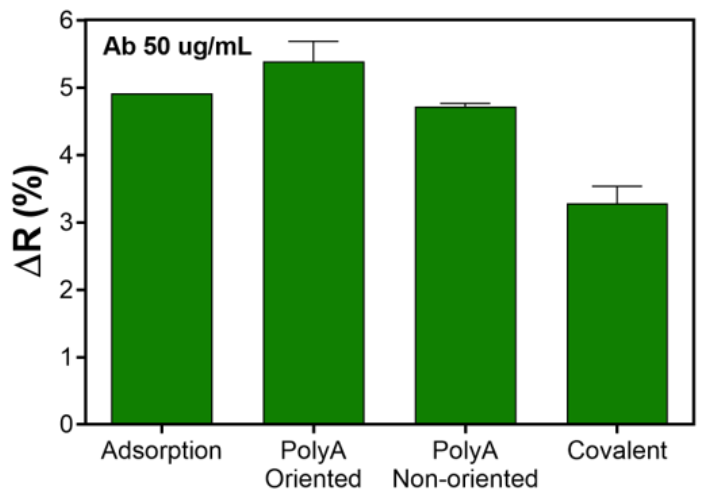

$\mathrm{B}$

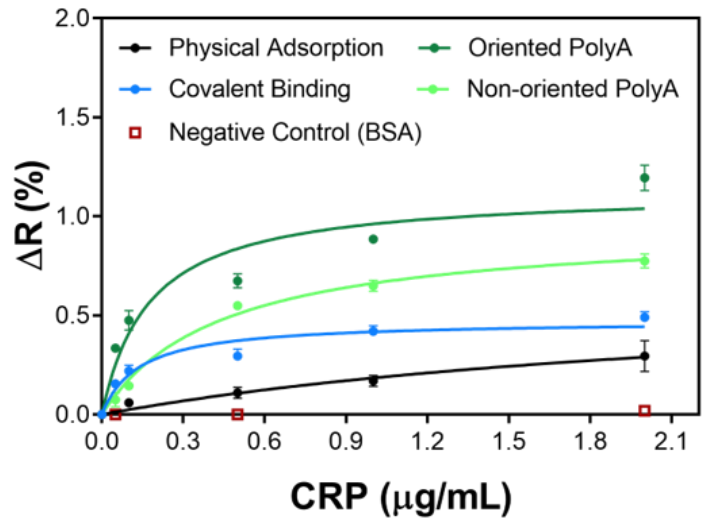

Figure 3. PolyA-Antibody assay. (A) Immobilization signals for the different approaches studied. (B) CRP calibration curves for each immobilization approach. Solid lines correspond to the nonlinear fit of the calibration curves. All data show mean \pm SD of triplicate measurements.

Stability and reusability of the polyA biosensor surface. The polyA-based sensor biofunctionalization is based on electrostatic interactions that do not involve chemical linkage as in the case of thiol chemisorption or covalent binding to a SAM. Therefore, it is important to study and evaluate the stability, reproducibility, and reusability of the biosensor when using this approach. Regeneration of the biorecognition layer, i.e. releasing of the captured target biomarker, was tested for both DNA and antibodies taking into account the possibility to perform repeated detection cycles with the same efficiency and sensitivity. Nucleic acid regeneration was achieved with 50\% formamide solution, which disrupts hybridization at RT. ${ }^{2}$ Repeated measurements were performed over 25 hybridization/regeneration cycles with signal variations of less than $3 \%$. On the other hand, antibody-antigen interaction was disrupted by $\mathrm{pH}$ change, using low-concentration acidic solution $(5 \mathrm{mM}$ $\mathrm{HCl})$. The sensor signal was monitored to effectively return to baseline and new measurements were performed over approximately 15 cycles before observing a significant decrease of the detection signal (62\%) (Figure 4A). This reduction of the signal can be attributed to a loss of antibody recognition capability due to the repetitive exposure to acidic $\mathrm{pH}$ conditions, rather than affecting the polyA-Ab immobilization stability. In general, for both DNA probes and antibodies, the polyAbiofunctionalization strategy showed a coefficient of variation (CV) between $3-12 \%$, which is below the $15 \%$ maximum limit recommended for analytical methods. ${ }^{25}$ In addition to the stability and reproducibility studies, we briefly investigated the possibility to recycle the sensor surface in situ, that is, the capability of removing the immobilized probes, cleaning the sensor surface, and subsequently generating a new polyA layer with a new or different probe. Thereby, we could perform different assays on the same sensor surface without replacement of the gold sensor chip, saving time and resources. To that end, we took advantage of the relatively weak interaction of adenine with gold compared to chemical binding. Strong acidic medium should break the hydrogen bonds as well as electrostatic interactions between adenine tail and the metal. In Figure 4B, we show the effect of $3 \mathrm{M} \mathrm{HCl}$ solution in a polyADNA functionalized biosensor. The strong acid solution is capable of almost completely remove the polyA-DNA layer within a few minutes. After, new in situ immobilization of polyA-DNA probe showed similar results to previous one, and we were able to hybridize a target sequence with practically the same efficiency. Although further improvement on the surface recycling should be done, these results open the opportunity for a faster and cost-effective operation of the biosensor, greatly reducing the number of sensor chips employed, contributing to generate less environmental waste as well as the time of the analyses.
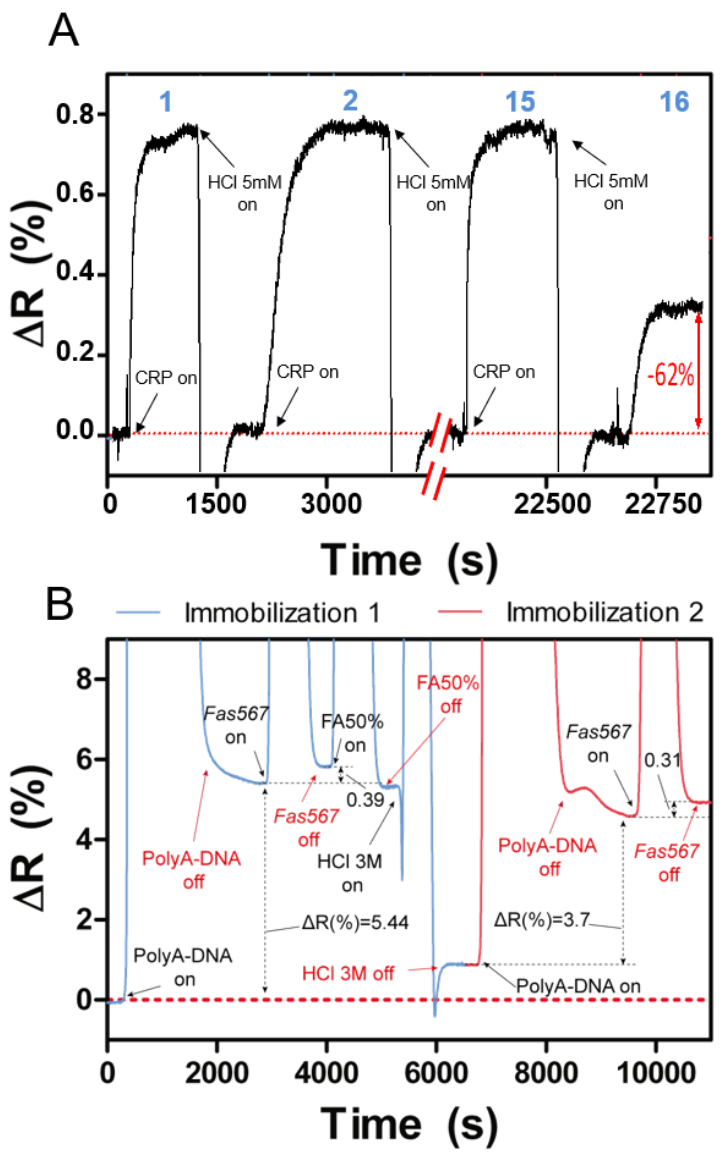

Figure 4 Reproducibility and recycling assessment of the onestep PolyA functionalization approach. (A) SPR sensorgram showing 16 detection cycles of CRP on a PolyA-Ab modified gold sensor chip. (B) SPR sensorgram showing two consecutive polyA-DNA immobilizations after the recycling of the gold sensor surface with $3 \mathrm{M} \mathrm{HCl}$. 
A

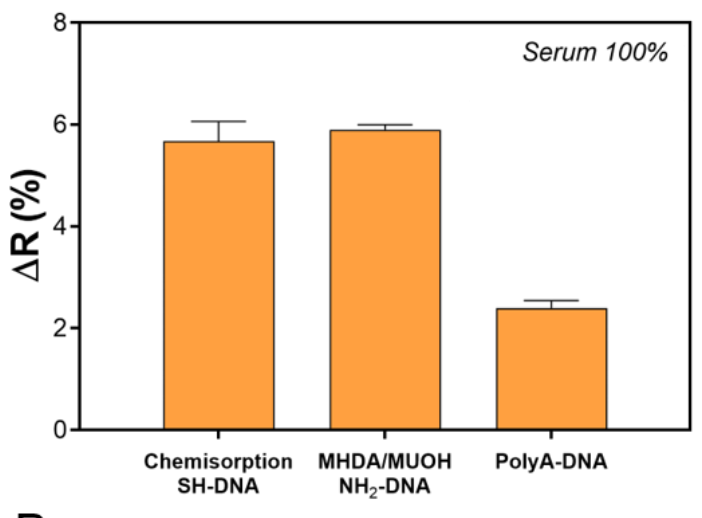

B

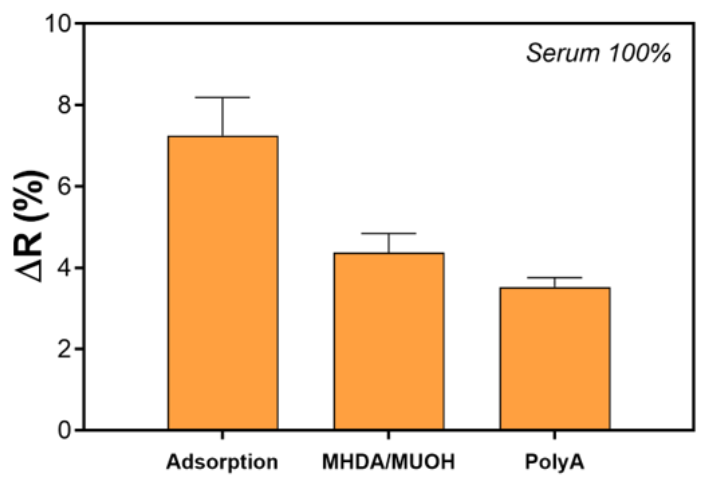

Figure 5. Assessment of the antifouling effect of the one-step polyA immobilization approach for serum $100 \%$. (A) Nonspecific binding on the DNA-probe immobilization strategies; (B) Nonspecific binding on the antibody immobilization strategies.

Antifouling test of the polyA based strategy. Finally, the fouling properties of this new immobilization approach were assessed. Serum is the most widely explored biofluid in clinical analysis. In the blood stream, there are many circulating biomarkers like soluble proteins, DNA and RNA, whose detection and precise quantification can inform about specific diseases or clinical conditions. However, direct evaluation of undiluted blood serum in SPR remains challenging and is still a not well-solved problem in direct label-free evaluation. The different molecules in serum matrix can interact with the sensor surface producing high background signals that hinder the specific biosensor response from the protein and nucleic acid targets or lead to false positive results. Gold sensor surfaces coated with adenine oligos have proven to be resistant to nonspecific binding of unwanted nucleic acid adsorption and proteins (i.e BSA) even at low densities due to the wide coverage area on behalf of the grafted polyA blocks (Figures 2B and 3B). This characteristic could provide an advantage in obtaining a suitable low-fouling monolayer for non-purified real sample analysis. To test our polyA-DNA and polyA-Ab monolayers, we compared their fouling resistance with SH-DNA, adsorbed antibodies, and alkanethiol SAMs. We flowed over the different monolayers a sample containing undiluted serum (serum 100\%). As can be observed in Figure 5A, the DNA monolayer coated with polyA blocks presented a high resistance to unwanted non-specific adsorptions from the matrix components of the serum sample $(\Delta \mathrm{R}(\%)=2.4)$, showing a decrease in the background signal of $41 \%$ with regard to the surface coatings based on thiol-chemistry, either using SHDNA probes or MHDA/MUOH alkanethiols $(\Delta \mathrm{R}(\%) \sim 6)$. These results evidenced the great fouling properties provided by the polyA-DNA monolayer. Similar antifouling properties were obtained with the polyA-Ab monolayer $(\Delta \mathrm{R}(\%)=3.5)$, minimizing the serum background signal by $52 \%$ and $20.5 \%$ compared to the antibody monolayers generated by physical adsorption and MHDA/MUOH, respectively (Figure 5B). Interestingly, the latter exhibited different fouling properties for DNA and antibody, showing less background signal in the antibody monolayer. This could be explained by the different electrostatic or hydrophobic forces of DNA and antibody with the soluble components of serum. Contrary, polyA-DNA monolayers showed higher fouling resistance than polyA-Ab ones. This phenomenon could be explained by the higher coverage obtained by the polyA-DNA, which was further increased by the combination of two polyA-lengths. Additional experiments need to be performed combining different polyA lengths conjugated with the antibody to maximize the gold sensor surface coverage. Overall, these results evidence the adequate anti-fouling properties provided by our one-step polyA strategy for both, proteins and DNA.

\section{CONCLUSIONS}

Diagnostic tools offering a multimarker detection can accelerate the implementation of precision diagnostics in the clinic. The simultaneous analysis of biomarkers based on soluble proteins and nucleic acids will stimulate the creation of more accurate biomarker panels that can inform about the molecular condition of patients. While technological advances in optical biosensing have boosted record levels of sensitivity, there is still an unmet need for direct and accurate surface chemistry approaches that benefit both, nucleic acid and protein detection. We have presented an innovative methodology for the efficient functionalization of gold surfaces in one step that is equally beneficial for nucleic acid and protein-based detection assays. Our approach allows the direct, in situ immobilization of DNA probes and antibodies in a single step, showing surface coverages comparable to the conventional multi-step methods. The methodology can accurately control the bioreceptor density, providing opportunities to generate biosensors that can detect analytes with more precision while avoiding steric hindrance repulsion forces. The results show excellent biosensor performances, obtaining competitive LODs for both, DNA and protein detection. We have demonstrated that the reproducibility of polyA biosensors can be maintained throughout 15-25 detection cycles with no loss in detection efficiency. Additionally, due to the non-covalent interaction of polyA-blocks with gold surfaces, we have proposed a surface recycling protocol, allowing the generation of a new bioreceptor monolayer without the need of the gold sensor chip replacement. This advantage places this strategy as a time and cost-effective methodology as well as environmentally friendly. Finally, the antifouling properties were demonstrated by assessing the background signal produced by undiluted serum. Our results confirmed that polyA based monolayers can reduce the background signal produced by the presence of other molecules in the sample by up to $50 \%$ without the employment of additional blocking agents. Further optimization of the methodology by surfactant addition or PEG molecules may minimize the background signals close to the system noise. These results highlight the one-step polyA immobilization as an exceptional and straightforward functionalization strategy that could eventually be used for the development of multiana- 
lyte biosensors for real sample evaluation, such as the socalled liquid biopsies, opening the frontier for more informative and accurate diagnostic tests that can help in precision medicine approaches.

\section{ASSOCIATED CONTENT}

\section{Supporting Information}

The Supporting Information is available free of charge on the ACS Publications website.

Details of reagents and buffers, thiol-DNA and Amine-DNA

probes immobilization methodology, and additional results for the validation of the methodology (PDF)

\section{AUTHOR INFORMATION}

\section{Corresponding Author}

*cesar.sanchez.huertas@rmit.edu.au.

\section{Author Contributions}

The manuscript was written through contributions of all authors. / All authors have given approval to the final version of the manuscript. / \$These authors contributed equally.

\section{ACKNOWLEDGMENT}

This work has made use of the Biodeposition and Biodetection Unit (U4) from ICTS NANBIOSIS partially supported by MICINN/FEDER (FICTS- 1420- 27 selected by MICINN). ICN2 is the recipient of Grant SEV-2017-0706 from the "Severo Ochoa Centers of Excellence" Program of Spanish Ministry of Science. Dr Cesar S. Huertas is a recipient of an RMIT Vice Chancellor's Postdoctoral Fellowship.

\section{REFERENCES}

(1) Borrebaeck, C. A. K. Precision Diagnostics: Moving towards Protein Biomarker Signatures of Clinical Utility in Cancer. Nature Reviews Cancer. Nature Publishing Group March 1, 2017, pp 199-204. https://doi.org/10.1038/nrc.2016.153.

(2) Huertas, C. S.; Calvo-Lozano, O.; Mitchell, A.; Lechuga, L. M. Advanced Evanescent-Wave Optical Biosensors for the Detection of Nucleic Acids: An Analytic Perspective. Front. Chem. 2019, 7. https://doi.org/10.3389/fchem.2019.00724.

(3) Soler, M.; Huertas, C. S.; Lechuga, L. M. Label-Free Plasmonic Biosensors for Point-of-Care Diagnostics: A Review. Expert Rev. Mol. Diagn. 2019, 19 (1), 71-81 https://doi.org/10.1080/14737159.2019.1554435.

(4) Han, X.; Liu, K.; Sun, C. Plasmonics for Biosensing. Materials $\begin{array}{lllll}\text { (Basel). } & \mathbf{2 0 1 9}, & 12 & \text { (9), } & 1411 .\end{array}$ https://doi.org/10.3390/ma12091411.

(5) Mauriz, E.; García-Fernández, M. C.; Lechuga, L. M. Towards the Design of Universal Immunosurfaces for SPR-Based Assays: A Review. TrAC - Trends in Analytical Chemistry. Elsevier B.V. May 2016, pp 191-198. https://doi.org/10.1016/j.trac.2016.02.006.

(6) Wong, L. S.; Khan, F.; Micklefield, J. Selective Covalent Protein Immobilization: Strategies and Applications. Chem. Rev. 2009, 109 (9), 4025-4053. https://doi.org/10.1021/cr8004668.

(7) Bergström, G.; Mandenius, C. F. Orientation and Capturing of Antibody Affinity Ligands: Applications to Surface Plasmon Resonance Biochips. Sensors Actuators, B Chem. 2011, 158 (1), 265-270. https://doi.org/10.1016/j.snb.2011.06.017.

(8) Cho, I. H.; Paek, E. H.; Lee, H.; Kang, J. Y.; Kim, T. S.; Paek, S. H. Site-Directed Biotinylation of Antibodies for Controlled Immobilization on Solid Surfaces. Anal. Biochem. 2007, 365 (1), 14-23. https://doi.org/10.1016/j.ab.2007.02.028.

(9) Soler, M.; Estevez, M. C.; Alvarez, M.; Otte, M. A.; Sepulveda, B.; Lechuga, L. M. Direct Detection of Protein Biomarkers in Human Fluids Using Site-Specific Antibody Immobilization Strategies. Sensors (Switzerland) 2014, 14 (2), 2239-2258. https://doi.org/10.3390/s140202239.

Brogan, K. L.; Wolfe, K. N.; Jones, P. A.; Schoenfisch, M. H. Direct Oriented Immobilization of $\mathrm{F}\left(\mathrm{Ab}^{\prime}\right)$ Antibody Fragments on Gold. Anal. Chim. Acta 2003, 496 (1-2), 73-80. https://doi.org/10.1016/S0003-2670(03)00991-7.

Huertas, C. S.; Calvo-Lozano, O.; Mitchell, A.; Lechuga, L. M. Advanced Evanescent-Wave Optical Biosensors for the Detection of Nucleic Acids: An Analytic Perspective. Front. Chem. 2019, 7. https://doi.org/10.3389/fchem.2019.00724.

Kimura-Suda, H.; Petrovykh, D. Y.; Tarlov, M. J.; Whitman, L. J. Base-Dependent Competitive Adsorption of Single-Stranded DNA on Gold. J. Am. Chem. Soc. 2003. https://doi.org/10.1021/ja035756n.

(13) Schreiner, S. M.; Hatch, A. L.; Shudy, D. F.; Howard, D. R.; Howell, C.; Zhao, J.; Koelsch, P.; Zharnikov, M.; Petrovykh, D. Y.; Opdahl, A. Impact of DNA-Surface Interactions on the Stability of DNA Hybrids. Anal. Chem. 2011. https://doi.org/10.1021/ac200814y.

Sohreiner, S. M.; Shudy, D. F.; Hatoh, A. L.; Opdahl, A.; Whitman, L. J.; Petrovykh, D. Y. Controlled and Efficient Hybridization Achieved with DNA Probes Immobilized Solely through Preferential DNA-Substrate Interactions. Anal. Chem. 2010, 82 (7), 2803-2810. https://doi.org/10.1021/ac902765g. Aviñó, A.; Jorge, A. F.; Huertas, C. S.; Cova, T. F. G. G.; Pais, A.; Lechuga, L. M.; Eritja, R.; Fabrega, C. Aptamer-Peptide Conjugates as a New Strategy to Modulate Human $\alpha$-Thrombin Binding Affinity. Biochim. Biophys. Acta - Gen. Subj. 2019. https://doi.org/10.1016/j.bbagen.2019.06.014.

(16) Soler, M.; Estevez, M. C.; Villar-Vazquez, R.; Casal, J. I.; Lechuga, L. M. Label-Free Nanoplasmonic Sensing of TumorAssociate Autoantibodies for Early Diagnosis of Colorectal Cancer. Anal. Chim. Acta 2016 https://doi.org/10.1016/j.aca.2016.04.059.

(17) Jabbari, S.; Dabirmanesh, B.; Arab, S. S.; Amanlou, M.; Daneshjou, S.; Gholami, S.; Khajeh, K. A Novel Enzyme Based SPR-Biosensor to Detect Bromocriptine as an Ergoline Derivative Drug. Sensors Actuators, B Chem. 2017. https://doi.org/10.1016/j.snb.2016.08.165.

(18) Huertas, C. S.; Carrascosa, L. G.; Bonnal, S.; Valcárcel, J.; Lechuga, L. M. Quantitative Evaluation of Alternatively Spliced MRNA Isoforms by Label-Free Real-Time Plasmonic Sensing. Biosens. Bioelectron. 2016, 78, 118-125. https://doi.org/10.1016/j.bios.2015.11.023.

(19) Huertas, C. S.; Bonnal, S.; Soler, M.; Escuela, A. M.; Valcárcel, J.; Lechuga, L. M. Site-Specific MRNA Cleavage for Selective and Quantitative Profiling of Alternative Splicing with LabelFree Optical Biosensors. Anal. Chem. 2019, 91 (23), 1513815146. https://doi.org/10.1021/acs.analchem.9b03898.

(20) Opdahl, A.; Petrovykh, D. Y.; Kimura-Suda, H.; Tarlov, M. J.; Whitman, L. J. Independent Control of Grafting Density and Conformation of Single-Stranded DNA Brushes. Proc. Natl. Acad. Sci. 2007, 104 (1), 9-14. https://doi.org/10.1073/pnas.0608568103.

(21) Ivanov, K. I.; Bašić, M.; Varjosalo, M.; Mäkinen, K. One-Step Purification of Twin-Strep-Tagged Proteins and Their Complexes on Strep-Tactin Resin Cross-Linked with Bis(Sulfosuccinimidyl) Suberate (BS3). J. Vis. Exp. 2014, No. 86. https://doi.org/10.3791/51536.

(22) Flor, A. C.; Williams, J. H.; Blaine, K. M.; Duggan, R. C.; Sperling, A. I.; Schwartz, D. A.; Kron, S. J. DNA-Directed Assembly of Antibody-Fluorophore Conjugates for Quantitative Multiparametric Flow Cytometry. ChemBioChem 2014, 15 (2), 267-275. https://doi.org/10.1002/cbic.201300464.

(23) Schmidt, C.; Robinson, C. V. A Comparative Cross-Linking Strategy to Probe Conformational Changes in Protein Complexes. Nat. Protoc. 2014, 9 (9), 2224-2236. https://doi.org/10.1038/nprot.2014.144.

(24) Dirksen, A.; Dirksen, S.; Hackeng, T. M.; Dawson, P. E. Nucleophilic Catalysis of Hydrazone Formation and Transimination: Implications for Dynamic Covalent Chemistry. J. Am. Chem. Soc. 2006, 128 (49), 15602-15603. https://doi.org/10.1021/ja067189k.

(25) Wood, R. How to Validate Analytical Methods. TrAC - Trends Anal. Chem. 1999, $18 \quad(9-10)$, 624-632. https://doi.org/10.1016/S0165-9936(99)00150-8. 


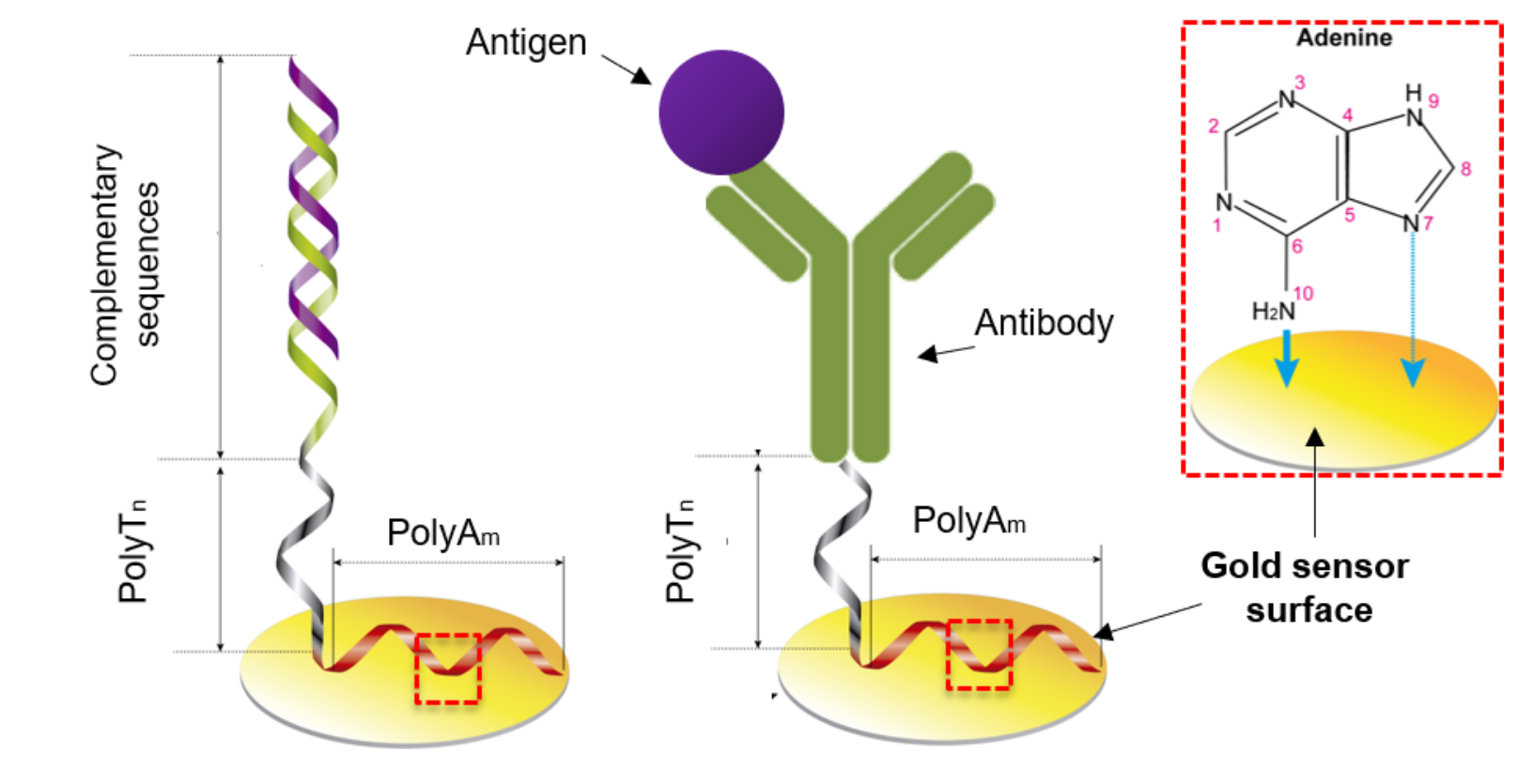

